\title{
Mobius Syndrome
}

National Cancer Institute

\section{Source}

National Cancer Institute. Mobius Syndrome. NCI Thesaurus. Code C84893.

A very rare congenital syndrome affecting the sixth and seventh cranial nerves. It is

characterized by facial paralysis leading to lack of facial expression and the inability to move the eyes laterally. 